\title{
L'EUROPE PARALYSÉE D'EFFROI - LA CRISE DE L'UNION EUROPÉENNE À LA LUMIÈRE D'UNE CONSTITUTIONNALISATION DU DROIT INTERNATIONAL PUBLIC
}

\author{
Jürgen Habermas et al. \\ Presses Universitaires de France | Cités \\ $2012 / 1-n^{\circ} 49$ \\ pages 131 à 146
}

ISSN 1299-5495

Article disponible en ligne à l'adresse:

http://www.cairn.info/revue-cites-2012-1-page-131.htm

Pour citer cet article :

Habermas Jürgen et al., « L'Europe paralysée d'effroi - La crise de l'Union européenne à la lumière d'une constitutionnalisation du droit international public »,

Cités, 2012/1 n 49, p. 131-146. DOI : 10.3917/cite.049.0131

Distribution électronique Cairn.info pour Presses Universitaires de France.

(C) Presses Universitaires de France. Tous droits réservés pour tous pays.

La reproduction ou représentation de cet article, notamment par photocopie, n'est autorisée que dans les limites des conditions générales d'utilisation du site ou, le cas échéant, des conditions générales de la licence souscrite par votre établissement. Toute autre reproduction ou représentation, en tout ou partie, sous quelque forme et de quelque manière que ce soit, est interdite sauf accord préalable et écrit de l'éditeur, en dehors des cas prévus par la législation en vigueur en France. II est précisé que son stockage dans une base de données est également interdit. 


\title{
GRAND ARTICLE INÉDIT
}

\author{
L'Europe paralysée d'effroi - La crise de l'Union européenne \\ à la lumière d'une constitutionnalisation \\ du droit international public ${ }^{1}$. \\ JÜRGEN HABERMAS
}

À court terme, la crise actuelle monopolise toute l'attention. Mais par-delà ceci, les acteurs politiques ne devraient pas oublier les défauts de construction qui sont au fondement de l'union monétaire et qui ne pourront pas être levés autrement que par une union politique adéquate. Il manque à l'Union européenne les compétences nécessaires à l'harmonisation des économies nationales, qui connaissent des divergences drastiques dans leurs capacités de compétition.

Depuis que le temps de l'embedded capitalism est révolu et que les marchés globalisés de la politique s'évanouissent, il devient de plus en plus difficile à tous les États de l'OCDE de stimuler la croissance économique et de garantir simultanément une répartition un tant soit peu juste des revenus ainsi que la sécurité sociale de la majorité de la population. Suite à la décision de la part de l'administration Reagan de libérer les taux de change, ce problème a été passagèrement désamorcé par l'acceptation d'une inflation croissante. Étant donné que cette stratégie entraîne des coûts élevés, les

1. Je remercie Armin von Bogdandy, Claudio Franzius et Christoph Möllers pour leurs conseils critiques.

Cités 49, Paris, Puf, 2012 
gouvernements utilisent de plus en plus l'échappatoire des participations aux budgets publics financées par le crédit. La crise financière qui dure depuis 2008 a également figé le mécanisme de l'endettement étatique aux frais des générations futures. Pour le moment, on ne voit pas comment les politiques d'austérité sévères - de toute façon difficiles à imposer en politique intérieure - pourraient se réconcilier sur la longue durée avec un niveau supportable de sécurité sociale.

Pendant ce temps et dans ces circonstances, le déséquilibre entre les impératifs du marché et la puissance régulatrice de la politique a été identifié comme étant le véritable défi. Au sein de la zone euro, un "gouvernement économique » vaguement espéré devrait redonner une force neuve au pacte de stabilité depuis longtemps évidé. Mais les représentations d'un "fédéralisme exécutif » d'un type particulier ${ }^{1}$, qui sont en circulation actuellement, reflètent la crainte des élites politiques de transformer le projet européen, jusque-là pratiqué derrière des portes closes, en un combat d'opinion bruyant et argumenté, obligeant à se retrousser les manches, et qui serait largement public. Au vu du poids incommensurable et sans précédent des problèmes, on s'attendrait à ce que les politiciens, sans délai ni condition, mettent les cartes européennes sur table sans délai et prennent l'initiative d'éclairer de manière offensive la population sur la relation entre les coûts à court terme et l'utilité véritable, et donc sur la signification historique du projet européen. Pour pouvoir le faire, ils devraient surmonter leur peur des sondages sur l'état de l'opinion et faire confiance à la puissance de persuasion de bons arguments. Au lieu de cela, ils s'acoquinent avec un populisme qu'ils ont eux-mêmes favorisé par l'obscurcissement d'un thème complexe et mal-aimé.

Concernant le seuil entre l'unification économique et politique de l'Europe, la politique semble retenir son souffle et rentrer la tête dans les épaules. Pourquoi cette paralysie ? C’est une perspective engluée dans le xix ${ }^{e}$ siècle, la thèse connue du "no demos ", qui le suggère : il n'existerait pas de peuple européen; et donc une union politique digne de ce nom serait édifiée

1. Stefan Oeter, "Föderalismus und Demokratie", A. v. Bogdandy, J. Blast éd., Europäisches Verfassungsrecht, Springer, Heidelberg, 2010, p. 73-120, utilise cette expression dans un autre sens : "Dans le système de l'union européenne, les bureaucraties participantes se dégagent largement du contrôle de leurs parlements (nationaux) en déplaçant les problèmes décisionnels sur le plan de l'Union. Mais sur le plan européen ils ne sont soumis à aucun des contrôles politiques seulement comparable à celui des systèmes constitutionnels nationaux. ", p. 104. 
sur du sable1. À cette interprétation, je voudrais en opposer une autre: la fragmentation politique durable dans le monde et en Europe est en contradiction avec la croissance systémique d'une société mondiale multiculturelle, et elle bloque tout progrès dans la civilisation juridique constitutionnelle des relations de puissance étatiques et sociales ${ }^{2}$. Carl Schmitt a épié avec suspicion cette tendance de la civilisation et l'a décrite comme une dissolution de la «substance " du " politique ». Il voyait cette substance comme une capacité juridiquement constituée d'auto-affirmation de l'État, à laquelle ne doivent pas être fixées de limites normatives. Selon la lecture de Schmitt, cette substance avait encore pu se manifester au commencement des Temps modernes dans le combat des États souverains contre les ennemis extérieurs et intérieurs. Elle ne se serait décomposée qu'avec les révolutions constitutionnelles du XviII ${ }^{\mathrm{e}}$ siècle, et ce tout d'abord à l'intérieur de l'État ${ }^{3}$.

L'État constitutionnel transforme les citoyens privés en des citoyens démocratiques nationaux; il rejette la notion d'ennemis intérieurs et voit ses adversaires - même dans sa lutte contre des terroristes - uniquement comme des criminels. Seule la relation de l'État souverain envers son environnement extérieur avait été "préservée " des chaînes normatives de la domestication légale 4 . Il n'est pas nécessaire de partager le jugement de Carl Schmitt pour apprécier le contenu descriptif qui résulte de son diagnostic lorsque l'on reconduit le "politique ", en l'arrachant au brouillard de son aura hostile aux Lumières, au noyau de la signification même du pouvoir de formation des autorités constitutionnelles.

Dans le champ des relations internationales, ce n'est qu'après l'échec de la Société des Nations et depuis la fin de la Seconde guerre mondiale

1. Une humeur qui a été renforcée en son temps en Allemagne, de manière anticyclique avec le traité de Maastricht, par la réunification de la nation divisée, cf. par exemple H. Lübbe, Abschied vom Superstaat. Vereinigte Staaten von Europa wird es nicht geben [Adieu au super-État. Il n'y aura pas d'États unis européens], Siedler, Berlin, 1994.

2. N. Elias, La civilisation des mours, Paris, Calmann-Lévy, 1973 (Tome 1 de Sur le Processus de civilisation), développe avant tout ce concept de civilisation eu égard à l'accroissement des capacités d'autocontrôle psychosociologiques dans le processus de la modernisation.

3. Carl Schmitt, Le concept du politique, Duncker \& Humblot, Berlin, 1932-1969; La notion de politique, traduction française M.-L. Steinhauser, Clamnn-Lévy, 1972.

4. C'est dans ce contexte que s'inscrit la polémique de Schmitt sa vie durant contre la pénalisation de la guerre d'agression dans le droit public ; cf. C. Schmitt, Die Wendung zum diskriminierenden Kriegsbegriff [Le tournant vers le concept de guerre discriminatoire], 1938, Berlin, 1988. 
- avec la création des Nations Unies et le commencement de l'unification européenne - qu'un profond assujettissement des États guerriers aura ouvert à une "juridicisation » dans le droit public qui va au-delà des restrictions placées sur la souveraineté nationale par le droit international classique. Ces tendances, accélérées depuis la fin de la Guerre froide, marquent le processus de civilisation qui être décrit selon deux points de vue complémentaires. De manière immédiate, la domestication de la violence interétatique vise une pacification des relations entre les États ; mais médiatement, c'est-à-dire par-delà la maîtrise de la concurrence anarchique entre les puissances et la promotion de la coopération entre les États, cette pacification rend également possible l'édification de nouvelles procédures supranationales et des institutions de négociation et de prise de décisions politiques. Car c'est seulement au moyen de nouvelles capacités directrices de politique transnationales que les forces sociales et économiques libérées au niveau transnational peuvent aussi être maîtrisées. Citons ici, et en guise d'exemple, les contraintes systémiques qui agissent sans se préoccuper des frontières nationales - aujourd'hui avant tout celles du secteur bancaire global ${ }^{1}$.

Le dense réseau des organisations supranationales fait craindre depuis longtemps que la relation entre droits fondamentaux et démocratie, qui est assurée dans l'État national, ne soit détruite et que les souverains démocratiques ne soient dépossédés par des puissances exécutives autonomisées à travers le monde entier ${ }^{2}$. Dans cette inquiétude se mêlent deux questions différentes. Je ne peux pas ici, brièvement, prendre position sur la question empirique bien justifiée de la dynamique économique de la société mondiale qui a renforcée depuis des décennies un déficit démocratique ${ }^{3}$. Mais, à l'exemple de l'Union européenne, je voudrais me confronter à cette autre déclaration dont se nourrit avant tout le défaitisme politique, à savoir

1. D. Held, A. McGrew, Governing Globalization, Polity Press, Cambridge UK, 2002.

2. Cf. la critique de Ingeborg Maus, "Menschenrechte und ermächtigungsnormen internationaler Politik oder : der zerstörte Zusammenhang von Menschenrechten und Demokratie ", in : H. Brunkhorst, W. R. Köhler, M. Lutz-Bachmann éd., Recht auf Menschenrechte, Suhrkamp, 1999, p. 276-292 ; la même, "Verfassung oder Vertrag. Zur Verrechtlichung globaler Politik ", in : P. Niesen, B. Herborth éd., Anarchie der kommunikativen Freiheit, Suhrkamp, 2007, p. 350-382.

3. M. Zürn, M. Ecker-Ehrhard éd., Die Politisierung der Weltgesellschaft, Berlin, 2011 ; voir également D. Held, A. McGrew éd., The Global Transformation Reader, Polity Press, Cambridge, UK, 2000. 
l'affirmation qu'une transnationalisation de la souveraineté populaire ne serait pas possible sans une réduction du niveau de légitimation.

Toute communauté démocratique est faite de trois composantes : de la mise en commun horizontale de personnes de droit libres et égales, d'une organisation bureaucratique pour l'agir collectif et du médium d'intégration politique qu'est la solidarité citoyenne. Certes, ces composantes ne sont entièrement satisfaites que dans le cadre de l'État national ; mais au niveau européen, elles pourraient également entrer dans une nouvelle configuration qui n'amoindrie pas nécessairement le caractère démocratique de la nouvelle politique supranationale, l'Union européenne que je décrirai aujourd'hui. Les deux innovations remarquables reposent sur le fait que les États membres, qui conservent leur monopole de l'usage légitime de la force, se subordonnent eux-mêmes au droit supranational pour des raisons fonctionnelles (II) et partagent leur souveraineté avec l'ensemble des citoyens de l'union (III). Néanmoins, en vue de la crise actuelle, il faudrait alors également que le partage originel de souveraineté entre les citoyens de l'Union européenne et les peuples européens soit transposé en une répartition symétrique des compétences entre le Conseil et le Parlement, et une responsabilité tout aussi symétrique de la Commission envers le Conseil et le Parlement (IV). En conclusion, je reviendrai sur le problème de l'élargissement de la solidarité entre les citoyens de l'État national (V).

PREMIÈRE INNOVATION : LA SUBORDINATION

DES ÉTATS NATIONAUX AU DROIT SUPRANATIONAL

Avant que nous puissions reconnaître quelle légitimité manque encore aux décisions européennes, nous devons louer la qualité démocratique de la forme qu'a prise l'Union Européenne avec le traité de Lisbonne'. À cette fin, je distingue entre trois éléments constitutifs ${ }^{2}$ qui doivent être incarnés dans toute communauté (Gemeinwesen) démocratique :

- l'association des personnes juridiques qui se réunissent dans un espace précis pour former une association de citoyens libres et égaux, en ce

1. I. Pernice, "Verfasungsverbund", in : Franzius, Mayer, Neyer, op. cit., 2010, p. $102-119$.

2. H. Brunkhorst, «A Polity without a State? European Constitutionalism between Evolution and Revolution ", in : E. O. Erikson, J. E. Fossum, A. J. Menendez éd., Developping a Constitution for Europe, Routledge, Londres, 2004 ; le même, State and Constitution : a Reply to Scheuerman, Constellations 15, 2008, p. 493-501. 
qu’ils s'aménagent des droits réciproques garantissant à chacun la même autonomie privée et citoyenne ;

- la répartition des compétences juridiques dans le cadre d'une organisation qui garantit par des moyens administratifs la capacité d'agir collective des citoyens associés; et,

- la solidarité citoyenne comme le médium d'intégration étatique ou supra-étatique qui est nécessaire à la formation d'une volonté politique commune et donc à l'engendrement communicationnel du pouvoir démocratique et à la légitimation de l'autorité publique'.

Ces trois composantes ne deviennent congruentes qu'au niveau national, que ce soit sous forme d'un État unitaire ou fédéral.

Au niveau national, l'autorité gouvernementale est programmée de telle sorte, par la procédure démocratique et dans la grammaire des lois générales, que les citoyens puissent exercer leur domination sur les organes législatifs, exécutifs et juridiques. Les citoyens d'une communauté [Gemeinwesen] démocratique ne se soumettent pas seulement factuellement au droit, parce que l'État menace de sanctions; ils ont l'option d'accepter le droit comme "juste " ou légitime parce qu'il a été institué démocratiquement. Soumettre l'exercice de l'autorité politique à la législation démocratique conduit à civiliser la violence, dans la mesure où l'exécutif choisi par le peuple, bien que disposant des moyens encasernés de la violence, doit respecter la constitution et la loi. Mais alors qu'au niveau national, les institutions qui posent et imposent le droit sont les organes d'un même État, dans l'Union européenne l'établissement et l'imposition du droit se déroulent sur différents niveaux. Au premier coup d'œil, il semble en aller de même dans les États fédéraux. Dans le système à plusieurs niveaux de la République fédérale allemande, la loi fédérale s'impose également à la loi des Länder, tandis que les gouvernements des Länder conservent néanmoins la haute main sur la police (même si ce n’est pas sur l'armée

1. Les trois composantes sont des éléments constitutifs d'un système de l'agir politique. Elles se rapportent à

- la constitution d'une communauté de personnes de droit,

- à l'habilitation à l'agir collectif et,

- à un horizon partagé du monde vécu au sein duquel peut se constituer communicationnellement une volonté collective.

Cette conceptualisation ne doit cependant pas porter préjudice à une analyse de science politique orientée exclusivement sur la théorie de l'agir. L'opinion politique communique dans la langue du droit, qui circule dans l'ensemble de la société, à propos de l'organisation étatique, et au moyen de tous les autres systèmes fonctionnels de la société. 
fédérale). Mais il y a une différence décisive entre le système national fédéral et le système européen à plusieurs niveaux.

Dans l'Union européenne s'est établie une préséance du droit européen sur le droit des États membres, même si les organes de l'Union, à la différence des organes législatifs d'un État fédéral ne disposent pas de la compétence de décider en dernier ressort, laquelle s'exprime entre autres dans la compétence à modifier la constitution ${ }^{1}$. Même si les États membres ne se considèrent plus tout simplement comme "maîtres des traités ", c'est à l'unanimité qu'ils doivent souscrire à une modification de traité correcte. Cet arrangement déplace les poids dans la relation entre le pouvoir de sanction étatique et le droit. En exerçant ses compétences législatives et juridiques, l'Union européenne lie les États membres en tant qu'organes exécutants sans disposer de leurs potentiels de sanction. Ce qui veut dire que les monopolistes du pouvoir étatique peuvent être mobilisés pour accomplir le droit européen qui doit être réinvesti nationalement.

Mais comment faut-il comprendre la préséance du droit européen ? Car, même si l'Union ne dispose pas de la compétence ultime de décision, et manque de ce que la jurisprudence allemande a appelé "KompetenzKompetenz ", la subordination, effective dans les faits, du droit national au droit de l'Union ne peut s'expliquer que par la hiérarchisation usuelle entre droit fédéral et droit des Länder, ou entre droit constitutionnel et droit secondaire. Des professeurs allemands de droit parlent d'une "préséance d'application ${ }^{2}$ " fondée fonctionnellement ou d'une " efficacité » du droit européen ${ }^{3}$ ». Mais comment doit-on pouvoir fonder une " préséance d'application" si la Communauté Européenne ne peut prétendre à une "préséance de validité » sur les systèmes juridiques nationaux ? Même le très eurosceptique tribunal fédéral constitutionnel de Karlsruhe ne se prévaut, dans ses décisions sur le traité de Maastricht et de Lisbonne, que d'une présomption de validité des constitutions nationales par rapport à la juridiction européenne. Dans l'interprétation des traités européens, les tribunaux nationaux ne sont pas autorisés à contrôler les limites du transfert de droits de souveraineté nationaux au niveau européen, mais ils

1. Chr. Calliess, Die neue Europäische Union nach dem Vertrag von Lissabon, Tübingen, Mohr Siebeck, 2010, p. 84 s. et p. 352 s.

2. C. Franzius, Europäisches Verfassungsrechtsdenken, Tübingen, Mohr Siebeck, 2010, p. 42.

3. A.v. Bogdandy, "Grundprinzipien ", in : Bogdandy, Bast éd., Europäisches Verfassungsrecht, $2^{\mathrm{e}}$ éd., Springer, 2010, p. 38. 
sont en revanche autorisés à surveiller l'intangibilité des principes constitutionnels nationaux qui sont constitutifs pour l'édification démocratique - et relevant de l'État - de droit de chaque État membre considéré individuellement. Ils protègent ce qu'Alain Laquièze nomme leur "identité constitutionnelle ». Les conflits entre les cours de justice des deux niveaux ${ }^{1}$ reflètent une dépendance et une intrication complémentaires entre constitutions nationales et droit communautaire, qui ont incité Ingolf Pernice à décrire l'Union comme une "confédération constitutionnelle ${ }^{2}$ ". Ainsi la subordination du droit européen est de facto une conséquence du fait que deux sujets constituants distincts ont été impliqués depuis le départ : les peuples d'Europe et le citoyen européen ont coopéré en vue d'un but commun : la création d'une communauté supranationale.

Afin de penser cette communauté supranationale d'un genre nouveau, la précision négative - que l'Union ne saurait être définie ni comme une fédération d'États ni comme un État fédéral - ne saurait suffire. La position éminente que le traité de Lisbonne confère au Conseil Européen et au Conseil des ministres reflète le rôle historique des États membres en tant qu'initiateurs et forces motrices de l'unification européenne. À la différence de nombreuses constitutions nationales des XVIII ${ }^{e}$ et XIX ${ }^{e}$ siècles, la constitution de l'Union est l'œuvre des élites politiques. Tandis que jadis des citoyens révolutionnaires s'alliaient pour renverser d'anciens régimes, ce furent cette fois-ci des États, c'est-à-dire des acteurs collectifs, qui se sont assemblés à l'aide de traités internationaux dans le but de coopérer dans des champs politiques limités. Mais, au cours du processus d'unification, le poids s'est très nettement déplacé en faveur des citoyens européens ${ }^{3}$. L'organisation internationale a été transformée en une union politique de durée indéterminée. Avec l'introduction de la citoyenneté européenne,

1. ... que le tribunal constitutionnel espagnol aimerait résoudre sémantiquement à l'aide des concepts de primacia du droit européen et de supremacia du droit national, cf. Franzius, op. cit., 2010, p. 47.

2. I. Pernice, "Europäisches und nationales Verfassungsrecht ", VVDStRL 60, 2001, p. $149-193$.

3. À propos des cing étapes dans le développement constitutionnel de l'U.E, cf. la contribution de Jürgen Bast, in : Franzius, Mayer, Nyer, op. cit., 2010, p. 173-180. 
avec le référence explicite à un intérêt publique européen partagé, et avec la reconnaissance d'une personnalité juridique propre à l'Union, les traités sont devenus le fondement d'une communauté politique dotée d'une constitution propre.

En déplaçant l'analyse de l'interprétation à la reconstruction rationnelle, nous pouvons jeter un regard rétrospectif sur le développement du traité - comme si le résultat historiquement plus ou moins contingent avait été produit intentionnellement par une assemblée constituante normalement composée. Ce faisant, nous nous heurtons à une question à laquelle avait déjà été confronté James Madison en 1787 lors de la fondation des ÉtatsUnis d'Amérique : une fédération démocratique d'États membres peut-elle satisfaire aux conditions de légitimation démocratique, sans que le niveau national ne soit unanimement subordonné au niveau fédéral, comme dans un État fédéral ${ }^{1}$ ?

Pour répondre à cette question de la légitimation, nous devons premièrement identifier correctement les puissances constituantes. Déjà l'article I, alinéa 1, du Traité instituant la Constitution Européenne se réfère aux deux sujets, aussi bien aux citoyens qu'aux États européens². Même si cette Convention de l'année 2004 a échoué, le Traité de Lisbonne qui est valide soutiens la thèse selon laquelle la souveraineté est " partagée $e^{3}$ " entre citoyens et États, pour la simple raison que lors de modifications du Traité constitutionnel, le Parlement est impliqué dans le processus et fait face au Conseil de Ministres comme un organe de rang égal dans le "processus législatif ordinaire ». Néanmoins, la scission du sujet constituant en « citoyens " et "États » exige une importante qualification. Au sein de la communauté politique de rang supérieur, les citoyens participent des deux côtés : de façon directe dans leur rôle de citoyens de l'Union, et de manière indirecte dans leur rôle de membre des peuples étatiques ${ }^{4}$. C'est pourquoi il est plus conséquent d'identifier non pas les États membres eux-mêmes, mais leurs peuples comme l'autre sujet du pouvoir constituant ${ }^{5}$.

1. The Debate on the Constitution, The Library of America, 1993, Part Two, p. 26-32.

2. "Inspirée par la volonté des citoyens et des États d'Europe de bâtir leur avenir commun, la présente Constitution établit l'Union européenne, à laquelle les États membres attribuent des compétences pour atteindre leurs objectifs communs. "

3. Calliess, op. cit., 2010, p. 71.

4. Je remercie Peter Niesen pour cette indication pertinente.

5. Bogdandy, op. cit., 2010, p. 64. Dans ce contexte, le renvoi à Kant est intéressant ; voir le comentaire de O. Eberl et P. Niesen, éd., Immanuel Kant, "Zum ewigen Frieden", Berlin, 2011, p. 166 : « Kant parle de la liberté des peuples, pas des États... Cela renvoie 
Reste encore à clarifier si nous devons reconnaître en ces sujets constituants « originels", comme le pensait James Madison, des citoyens d'États fondateurs qui ne sélèvent en citoyens de l'Union que par le processus de constitutionnalisation ; ou bien si nous reconnaissons en eux immédiatement les futurs citoyens de l'Union ${ }^{1}$.

Le choix entre ces deux alternatives malheureuses produirait encore un préjudice en faveur de l'attribution finale de l'autorité décisionnel ou bien à l'Union ou bien à ses États membres. Si nous voulons éviter de retomber sur l'alternative entre une confédération d'États et un État fédéral, il est recommandable d'introduire ces mêmes personnes (ou bien leurs représentants) en tant que sujets constituants en deux rôles différents, à savoir, dans le rôle des (futures) citoyens de l'Union et dans celui de citoyen d'un de ses États membres.

Ce qui compte, au sein d'un État-nation, comme une orientation vers le bien commun, se transforme au niveau européen en une orientation particulière, une généralisation des intérêts limités au peuple propre, qui peut entrer en conflit avec la généralisation à l'Europe entière des intérêts attendue par les citoyens de l'Union. De la sorte, les deux aspects du rôle des personnes constituantes pour la communauté constituée acquièrent une signification institutionnelle: au niveau européen, le citoyen doit simultanément, et en pesant du même poids, former son jugement et décider politiquement à la fois en tant que citoyen de l'Union et en tant qu'il appartient au peuple d'un État particulier qui appartient à l'Union.

QUE DÉCOULE-T-IL DE LA PARTITION DES PUISSANCES CONSTITUANTES POUR L'ÉDIFICATION LÉGITIME DE L'UNION ?

Bien entendu, l'expression de "souveraineté partagée » est ambiguë. La souveraineté du peuple, c'est-à-dire le " pouvoir qui émane du peuple » se

à ceci que ce qui importe à Kant est... la liberté des peuples dans le droit étatique et non pas la liberté des États dans le droit international. »

1. C'est en ce sens qu'Ingo Pernice, "Verfassungsverbund ", in Franzius, Mayer, Nyer, 2010, p. 102-118, défend la conception que sont transférés à l'Union européenne « originairement par les citoyens des États membres en commun " (p. 106) des droits de souveraineté. Il déduit de là que « la citoyenneté de l'Union est le statut politique commun qui se rapporte à l'Union et à sa légitimation, (statut) que les citoyennes et citoyens des États membres se sont donné en tant que citoyens de l'État à travers la constitution de l'Union Européenne »p. 108. 
ramifie et s'éparpille dés le début dans les flux communicationnels du législatif, de l'exécutif et du juridique, au sein de chaque communauté démocratiquement constituée. Le "peuple", en tant que tel, ne peut pas agir. Mais dans le contexte qui est le nôtre, c'est d'un autre partage de souveraineté dont il est question. Dans le cas de l'Union européenne, le partage du pouvoir constituante partage la souveraineté populaire à l'origine de la communauté à constituer et non pas seulement à la source de la communauté déjà constituée déjà. Cette division explique pourquoi l'Union européenne partage avec des États fédéraux le caractère d'un système à plusieurs niveaux, mais l'Union européenne ne doit pas être compris comme une sorte de république fédérale imparfaite. Même dans les Etats fédéraux, la répartition des compétences remonte en général à une habilitation limitée à des cas isolés des organes fédéraux par les États membres. Mais aussi longtemps que les citoyens d'une nation opèrent tous comme les sujets constituants de l'ensemble de l'État, ils n'établissent pas seulement une préséance du droit fédéral, mais ils conservent également, ou bien pour eux-mêmes (à travers des référents nationaux) ou pour les organes fédéraux constituants, l'habilitation à modifier la constitution ${ }^{1}$.

La figure de pensée de la souveraineté populaire « originellement partagée " ici évoquée inclut qu'il puisse exister au niveau européen une telle autorité constitutionnelle suprême. Les sujets constituants, en tant qu'ils appartiennent aux (futurs) États membres, sont certes prêts à transférer une partie des droits régaliens de leurs États déjà constitués à la nouvelle communauté ; mais ils ne le font qu'avec une réserve qui dépasse de loin la garantie par ailleurs habituelle de la structuration étatique partielle de la communauté fédérale. Par leur participation au processus constituant, les peuples européens s'assurent plutôt que leur propre État soit conservé, au sein de la communauté fédérale, dans la fonction garante de liberté d'un État de droit démocratique. La souveraineté partiellement conservée des États membres trouve son expression aussi bien dans l'article $48^{2}$ sur la procédure de révision ordinaire des traités et qui demande l'unanimité, que dans

1. Ce qui vaut aussi pour la Constitution fédérale suisse de 1998 (art. 192-194), bien que (d'après le préambule et l'art. 1 alinéa 1) la Confédération helvétique soit fondée simultanément par «le peuple suisse et les cantons ". Les organes de la fédération jouissent par ailleurs des prérogatives (art. 184-186) qui caractérisent la compétence compétente d'un État fédéral.

2. Traité sur l'Union européenne. Version consolidée en vigueur depuis le $1^{\text {er }}$ décembre 2009 (traité de Lisbonne). 
l'article 50, selon lequel «Tout État membre peut décider, conformément à ses règles constitutionnelles, de se retirer de l'Union. "

À mon sens, ces déviations du modèle connu de légitimation ne trahissent aucun déficit démocratique, au moins aussi longtemps que les deux sujets constituants, c'est-à-dire les citoyens de l'Union et les peuples européens, sont reconnus dans toutes les fonctions législatives comme des partenaires de même droit.

La souveraineté " partagée originaire » fournit un critère pour les besoins de légitimation d'une communauté politique désétatisée. Mais cela ne permet pas seulement de justifier les déviations par rapport au modèle de l'État fédéral, mais aussi de reconnaître les déficits démocratiques des traités européens existants. Aujourd'hui, nous sommes confrontés au défi de pouvoir redonner, au sein des pouvoirs politiques et des compétences législatives, l'égalité des droits et la relation symétrique que nous attribuons aux peuples européens et citoyens de l'Union lorsque nous reconstruisons rationnellement et contre-factuellement leurs rôles dans le procédé de fonder l'Union européenne. En conséquence, une stricte égalité entre le Conseil et le Parlement devrait être établie dans tous les champs politiques pertinents. La situation étrangement flottante de la Commission, à laquelle sont réservés certains droits d'initiative, est elle aussi inconséquente. Au lieu de cela, la Commission - à la différence d'un gouvernement fédéral devrait dépendre de manière symétrique du Parlement et du Conseil, et être responsable envers les deux. Plus important encore est le caractère totalement atypique du Conseil européen. C'est un organe dirigeant qui fixe les lignes directrices de la politique, mais ne possède ni de droit législatif ni de droit de donner des directives à la Commission.

Il existe une opposition étrange entre le pouvoir politique qui est concentré dans le Conseil européen et l'absence d'efficacité juridique de ses décisions. Pourvu de la légitimation indirecte de chefs de gouvernement choisis, un organe juridiquement non lié - qui doit certes prendre ses décisions à l'unanimité - exerce un pouvoir extraconstitutionnel considérable ${ }^{1}$. Le traité de Lisbonne à travers de l'intégration du Conseil européen dans la structure institutionnelle, était censé donner plus de capacité d'action à l'Union européenne; mais il en paye un prix élevé pour cela dans la forme du manque de légitimité des résolutions qui ont des grandes implications. Ce qui devient apparent, depuis la crise financière de 2008, dans

1. Bogdandy, op. cit., 2010, p. 44. De même Franzius, op. cit., 2010, p. 58. 
les décisions lourdes de conséquences de créer des " parapluies » fiscaux, et de nouvelles modalités d'harmonisation entre les gouvernements des États membres.

\author{
EN VUE DE MEILLEURES AVANCÉES \\ VERS UNE INTÉGRATION EUROPÉENNE PLUS COMPLÈTE, \\ NOUS DEVONS DÉCIDER ENTRE LE FÉDÉRALISME EXÉCUTIF \\ ET UNE DÉMOCRATIE SUPRANATIONALE PROPRE
}

Jusque-là nous avons parlé de deux des trois composantes constitutionnelles qui entrent dans une nouvelle constellation sur le niveau européen - l'expansion du cadre constitutionnel et la distribution des compétences dans un système à niveaux multiples Dès lors qu'une communauté constitutionnelle s'étend au-delà du noyau organisationnel d'un seul État, la troisième partie constitutive, la solidarité entre les citoyens qui sont prêts à s'engager les uns envers les autres, devrait dans une certaine mesure croître en même temps. L'ensemble des citoyens de l'Union ne peut partager effectivement la souveraineté avec les peuples des États membres qui continuent à monopoliser la violence que si la solidarité citoyenne de l'État subit une transformation de forme. D'après le scénario qui est le nôtre, je 143 propose, une solidarité citoyenne élargie, fut-elle plus abstraite, c'est-à-dire comparativement moins capable [Belastungsfähig], devrait à chaque fois inclure les membres des autres peuples européens. Ce n'est qu'alors que les citoyens de l'Union, qui élisent et contrôlent le Parlement de Strasbourg, sont à même de prendre part à la formation d'une volonté démocratique commune qui va au-delà des frontières nationales ${ }^{1}$. Certes, il est possible, dans le meilleur des cas, de stimuler par des moyens administratifs et juridiques la libéralisation d'orientations de valeurs, une disponibilité accrue à l'inclusion d'étrangers et une transformation adéquate d'identités collectives. Néanmoins il subsiste une interaction circulaire, se renforçant ou s'empêchant réciproquement, entre processus politiques et normes constitutionnelles d'une part, et le réseau de positions et de convictions culturelles et politiques d'autre part. Des loyautés anciennes s'affaiblissent, des solidarités nouvelles se développent, les traditions changent et les nations,

1. J. Habermas, «Ist die Herausbildung einer europäischen Identität nötig, und ist sie möglich ? [La formation d'une identité européenne est-elle nécessaire, et est-elle possible] ", in : Der Gespaltene Westen, Suhrkamp, 2004, p. 85-110. 
de même que tous les autres rapporteurs comparables, ne sont pas non plus des faits naturels.

L'extension supranationale de la solidarité des citoyens de l'État dépend, comme la crise actuelle permet de l'espérer, de processus d'apprentissage qui peuvent être stimulés par la perception de nécessités économiques et politiques. Car entre-temps, la ruse de la raison économique a mis en branle une communication dépassant les pays ${ }^{1}$; mais ce n'est que dans la poursuite d'une ouverture des opinions publiques nationales les unes aux autres que celle-ci pourra se condenser en un contexte communicationnel. Nous n'avons pas besoin de nouveaux média pour une transnationalisation des opinions publiques existantes, mais d'une pratique différente des média dominants existants. Ces derniers ne doivent pas seulement rendre présents et traiter les thèmes européens comme tels, mais simultanément rendre compte des diverses prises de position et controverses que les mêmes thèmes déclenchent dans les autres États membres.

Étant donné que jusque-là l'Union européenne a été essentiellement portée et monopolisée par les élites politiques, une dangereuse asymétrie en a résulté - une asymétrie entre la participation démocratique des peuples aux bénéfices que leurs gouvernements " en retirent " pour eux-mêmes sur la scène subjectivement éloignée de Bruxelles, et l'indifférence, voire l'absence de participation des citoyens de l'Union eu égard aux décisions de leur parlement à Strasbourg. Cette observation ne justifie cependant pas une substantialisation "du peuple» ou "de la nation». Seul le populisme de droite continue de projeter la caricature de grands sujets nationaux qui se ferment les uns aux autres et bloquent toute formation de volonté dépassant les frontières. En outre, après cinquante ans d'immigration de travail, les peuples européens, au vu de leur pluralisme ethnique croissant, langagier et religieux, ne peuvent plus être imaginés comme des unités culturelles homogènes ${ }^{2}$. De plus, l'Internet rend toutes les frontières poreuses. Parmi les vastes territoires de nos États-nations, il a fallu de tous temps commencer par installer l'horizon fluide d'un monde de la vie partagé sur de grands espaces et à travers des relations complexes, et le remplir par un

1. La Commission met en ligne les communiqués de presse de différents pays sur les thèmes important sous http://www.presseurop.eu/de.

2. K. Eder, "Europäische öffentlichkeit und multiple Identitäten - Das Ende des Volksbegriffs ?", in : C. Franzius, U. K. Preuss, éd., Europäische Öffentlichkeit, 2004, p. $59 \mathrm{~s}$. 
contexte communicationnel relevant de la société civile, avec son système circulatoire d'idées.

Il va sans dire que cela ne peut se faire que dans le cadre d'une culture politique partagée demeurant assez vague. Mais plus les populations nationales prennent conscience, et plus les média portent à la conscience, à quelle profondeur les décisions de l'Union européenne influent sur leur quotidien, plus croîtra l'intérêt qu'ils trouveront à faire également usage de leurs droits démocratiques en tant que citoyens de l'Union. Ce facteur d'impact est devenu tangible dans la crise actuelle de l'euro. Lorsque des problèmes de justice distributive apparaissent dans une communauté politique, les citoyens ne peuvent plus se satisfaire de la légitimation extérieure imposée qui a caractérisé l'Union européenne jusqu'à présent ; ils demandent une légitimation participative et active ${ }^{1}$. Selon cette logique, les citoyens nationaux - aujourd'hui devant subir des changements de répartition des charges au-delà des frontières nationales - veulent aussi influer démocratiquement, dans leur rôle de citoyen de l'Union, sur ce que leurs chefs de gouvernement négocient ou décident dans une zone constitutionnelle grise.

À l'inverse, nous constatons, du côté des gouvernements des tactiques dilatoires et, du côté des populations, un rejet populiste du projet européen dans son ensemble. Ce comportement autodestructeur s'explique par le fait que les élites politiques et les médias hésitent à tirer des conséquences raisonnables du projet constitutionnel. Sous la pression des marchés financiers s'est imposée la conviction que, lors de l'introduction de l'Euro, un présupposé économique essentiel du projet constitutionnel avait été négligé. L'Union européenne ne peut s’affirmer contre la spéculation financière - les analystes sont unanimes sur ce point - que si elle obtient les compétences politiques de guidage qui sont nécessaires pour garantir au moins dans le cœur de l'Europe, c'est-à-dire parmi les membres de la zone monétaire européenne, une convergence des développements économiques et sociaux des pays membres². Angela Merkel et Nicolas Sarkozy ont conclu un compromis entre le libéralisme économique allemand et l'étatisme français. Si je vois juste, ils cherchent à consolider le

1. F. W. Scharpf, Chr. Schmid, Regieren in Europa: Effektiv und Demokratisch?, Campus, 1999.

2. Concernant les possibilités juridiques d'une différenciation européenne intérieure, cf. D. Thym, "Variable Geometrie in der Europäische Union ", in Kadelbach, 2011, op. cit., p. 117-136. 
fédéralisme exécutif impliqué dans le traité de Lisbonne en une domination intergouvernementale du Conseil de l'Europe contraire au traité. Un tel régime permettrait de transférer les impératifs des marchés aux budgets nationaux sans aucune légitimation démocratique propre. Pour ce faire, il faudrait que des accommodements conclus dans l'opacité, et dépourvus de forme juridique, soient imposés à l'aide de menaces de sanctions et de pressions sur les parlements nationaux dépossédés de leur pouvoir. Les chefs de gouvernement transformeraient de la sorte le projet européen en son contraire : la première communauté transnationale démocratiquement légalisée deviendrait un arrangement particulièrement effectif, parce que voilé, d'exercice d'une domination post-démocratique.

L'alternative se trouve dans la continuation conséquente de la légalisation démocratique de l'Union européenne. Une solidarité citoyenne s'étendant à l'Europe entière ne peut pas se former si, entre les États membres, c'està-dire aux possibles points de rupture, se consolident structurellement des inégalités sociales entre nations pauvres et riches. L'Union doit garantir ce que la Loi fondamentale de la république fédérale allemande appelle (art. 106, alinéa 2) : "l'uniformité des conditions de vie ». Cette " uniformité " ne se rapporte qu'à une estimation des situations de vie sociales qui soit acceptable du point de vue de la justice distributive, et non pas à un nivellement des différences culturelles. Or, une intégration politique appuyée sur le bien être social est nécessaire pour que la diversité nationale et l'incomparable richesse culturelle du biotope de la "vieille Europe " puissent être protégées du nivellement au sein d'une globalisation à progression tendue.

Traduction de Denis Trierweiler, revue par Joseph Cohen et Sara Vigil 